\title{
EL HACHA PULIMENTADA DE JADE DE LA COLECCIÓN TUBINO (MUSEO MUNICIPAL, VALENCINA DE LA CONCEPCIÓN, SEVILLA)
}

\author{
A JADE POLISHED HAND-AXE FROM TUBINO'S COLECTION (VALENCINA \\ DE LA CONCEPCIÓN LOCAL MUSEUM, SEVILLA, SPAIN)
}

\author{
CARLOS P. ODRIOZOLA* \\ JUAN MANUEL VARGAS JIMÉNEZ** \\ JOSÉ MARÍA MARTÍNEZ-BLANES*** \\ LEONARDO GARCÍA SANJUÁN*
}

Resumen: En este trabajo se estudia un hacha pulimentada inédita excepcional depositada en el Museo de Valencina de la Concepción (Sevilla) en noviembre de 2010 y que formó parte de la colección personal de Francisco María Tubino y Oliva (1833-1888). Aunque la procedencia exacta de la pieza es desconocida, existen razones para pensar que podría proceder de algún yacimiento arqueológico de la provincia de Sevi1la. Mediante la aplicación de dos técnicas analíticas diferentes (XRD, DC $\mu R S)$, se identifica la pieza como jade-jadeíta de una pureza superior al $95 \mathrm{ml} \%$, lo que implica su procedencia de los Alpes. En las conclusiones se realiza una valoración de la significación de esta pieza en términos de contactos a larga distancia en la Prehistoria Reciente ibérica.

Palabras Clave: Prehistoria Reciente, Hacha Pulimentada, Jade, Espectroscopia $\mu$-Raman, Difracción de Rayos X, Intercambio de Larga Distancia, Comercio

\begin{abstract}
A polished axe donated to the Valencina de la Concepción Museum (Seville, Spain) in November 2010, and that had belonged to Francisco María Tubino y Oliva's (1833-1888) personal collection, is studied. Although the exact provenance of this object is unknown, there are reasons to think that it might have been found on some archaeological site of the Seville province. Through the application of two different analytical techniques (XRD, DC $\mu R S)$, this axe is identified as a jade-jadeite of purity above $95 \mathrm{ml} \%$, which necessarily implies it came from the Alps. In the conclusions a discussion of this artifact is made in terms of long distance contact in Iberian Late Prehistory.

Keywords: Late Prehistory, Polished Axe, Jade, $\mu$-Raman Spectroscopy, X-Ray Diffraction, Long Distance Exchange, Trade
\end{abstract}

* Departamento de Prehistoria y Arqueología de la Universidad de Sevilla. c/. María de Padilla, s/n. 41004-Sevilla. Correo-e: codriozola@us.es, lgarcia@us.es

** Museo de Valencina, Monográfico del Yacimiento Prehistórico. Correo-e: jmvargas66@gmail.com
*** Instituto de Ciencia de Materiales de Sevilla. Centro Mixto Universidad de Sevilla-Consejo Superior de Investigaciones Científicas.Correo-e: blanes@icmse.csic.es 


\section{INTRODUCCIÓN}

La pieza objeto de este estudio es un hacha de piedra verde pulimentada actualmente depositada en el Museo de Valencina, Monográfico del Yacimiento Prehistórico (Sevilla, España). Esta pieza fue donada a dicho Museo en Noviembre 2010 por sus hasta entonces propietarios, los herederos de F. M. Tubino y Oliva, al cumplirse el 150 aniversario del descubrimiento del tholos de La Pastora, y con motivo del congreso científico que se celebró para conmemorar dicho evento (García Sanjuán et al. 2012).

La pieza fue donada (gracias a las gestiones realizadas por María Teresa Ruiz Moreno, entonces delegada de cultura del Ayuntamiento de Valencina) por Mercedes y Rafael Tubino, descendientes de Francisco María Tubino y Oliva (San Roque, 1833-Sevilla, 1888) pionero de la arqueología española, y de acuerdo con los donantes, se trata de la única pieza arqueológica de la Colección Tubino que había persistido en la familia desde la desaparición de tan insigne personaje. Fue expuesta al público por primera vez con motivo de la celebración del Día Internacional de los Museos en mayo de 2011.

Formalmente, esta hacha muestra silueta lanceolada y sección oval con el filo curvo y el talón apuntado (fig. 1). Su factura es muy cuidada y el pulimentado de extremada finura al punto de conservar un buen brillo superficial. Junto al borde de corte y en una de sus caras se aprecia una pequeña rotura de aspecto reciente. Posee unas dimensiones máximas de 9,4 $\mathrm{cm}$ de longitud, $3,9 \mathrm{~cm}$ de anchura y $1,4 \mathrm{~cm}$ de grosor, siendo su peso total de $89 \mathrm{~g}$.

No se posee certeza documental sobre la procedencia de esta pieza. Es cierto que Tubino no la cita entre los materiales de La Pastora que él recogió o inventarió (Tubino y Oliva 1868a: 52), pero hay que tener en cuenta que tras el descubrimiento de La Pastora se produjo en la zona una intensa actividad exploratoria por parte de los buscadores de tesoros que frecuentaron el lugar y a los que hace referencia el propio Tubino (Tubino y Oliva, 1868b: 52). La reciente prospección geofísica de La Pastora y su entorno ha revelado la existencia de numerosas anomalías que salpican su túmulo, lo que ha sido puesto en conexión con dichos expolios (Vargas Jiménez et al. 2012: 135). Por tanto, no es posible descartar por completo que la pieza le llegara posteriormente a través de alguno de los aficionados y expoliadores locales, sin que ello quedara reflejado en sus publicaciones.

No parece esa la hipótesis más probable, no obstante, dado que en las referencias que acompañan a la primera comunicación sobre el descubrimiento de La
Pastora, el autor indica que durante sus exploraciones en las provincias de Sevilla, Cádiz y Huelva había recogido varias hachas procedentes de Sierra Morena y del término de Jerez de la Frontera (Cádiz) que, siempre de acuerdo con su propio relato, posteriormente habría entregado al Museo Arqueológico Nacional (MAN), a excepción de un hacha de 'jade' (Tubino y Oliva 1868a: 50). Hay que resaltar que entre los materiales entregados por F. M. Tubino al MAN, se encuentra un fragmento de hacha de jade recogido en las inmediaciones de El Pedroso (Sevilla) (Tubino y Oliva 1868a: 51). Por tanto, esto abre la posibilidad de que la pieza ahora donada al Museo de Valencina, y que siempre había estado en posesión de la familia Tubino, fuera el hacha de jade que F. M. Tubino decidió no donar al MAN. A pesar de que no existe constancia documental de la procedencia de esta excepcional pieza, los indicios del relato original de Tubino apuntan la posibilidad de que sea 'pareja' del fragmento de hacha de El Pedroso (Sevilla) y por tanto proceda de algún yacimiento arqueológico de esa zona.

El descubrimiento de La Pastora y los subsiguientes trabajos de F.M. Tubino sobre la Prehistoria andaluza (1868a; 1868b; 1876) coincidieron con el descubrimiento de los ajuares de las grandes construcciones megalíticas del Golfo de Morbihan (Francia), en los que destacan particularmente los espectaculares artefactos elaborados en piedra verde. Tal es el caso de las cuentas de collar y grandes hachas pulimentadas que, en el caso de las de Locmariaquer/Mané er Hroëck alcanzan 45,6 cm de longitud. Estas magníficas hachas fueron estudiadas por primera vez por el francés Damour (1865), quien basándose en estudios petrológicos identificó como jadeíta la materia prima de las hachas carnnacienses (usaremos este término como traducción literal del francés carnaceennes) proponiendo el macizo de Mont Viso como probable fuente de aprovisionamiento (Damour, 1881, citado en Petrequin et al., 2011b.) . Es conocido que F. M. Tubino no solo estaba al corriente de los descubrimientos que tenían lugar por esos años en Bretaña, sino que él mismo visitó la región y conoció sus más importantes sitios prehistóricos (Ruiz Moreno, 2012: 203), por lo que es muy probable que estuviera al corriente de los hallazgos de grandes hachas de piedra verde en los megalitos bretones.

El principal objetivo de este artículo es la caracterización precisa de la mineralogía, composición química y color del hacha de la Colección Tubino, utilizando técnicas arqueométricas, con el objeto de determinar si se trata de una pieza de jade. Posteriormente se hace una valoración de los resultados obtenidos en clave de 
Figura 1: Fotografía y dibujo del hacha $(15 / 26)$.

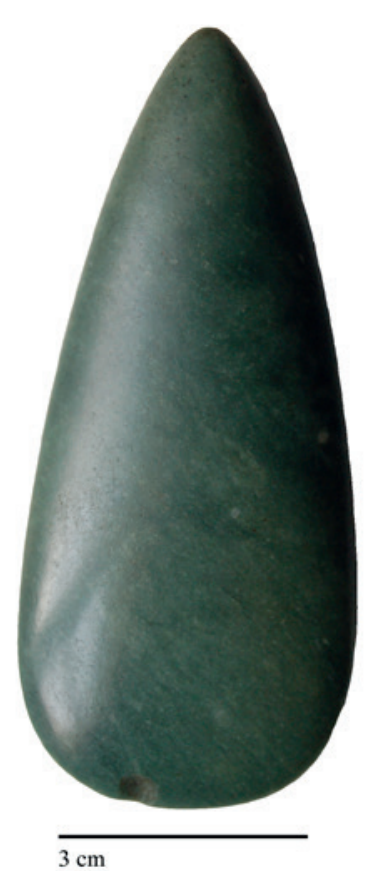

la rareza del objeto, su procedencia y de la distribución de piezas de jade conocidas en la península ibérica. El interés que esta pieza presenta es que, a pesar de su descontextualización, y a la imposibilidad de determinar su exacta procedencia, las circunstancias conocidas de su hallazgo y su historia permiten suponer que procede de algún yacimiento prehistórico de la provincia de Sevilla. La presencia de jade prehistórico en una región tan meridional es una importante noticia científica, ya que desde la última década del siglo XIX y primera del siglo XX, quedó establecido el origen alpino (concretamente del piedemonte de Mont Viso, el Valle Bulè y el macizo de Beigua) de las hachas prehistóricas pulimentadas de jade halladas en Europa Occidental (Petrequin et al. 2011b). Tras las numerosas investigaciones y publicaciones aparecidas desde entonces, tal atribución sigue no sólo vigente, sino consolidado como único posible para las hachas pulimentadas verdes neolíticas de jade (Ricq-de-Bouard et al. 1990; Ricq-de-Bouard y Fedele, 1993; D’Amico et al. 1995; Petrequin and Jeunesse, 1995, Petrequin et al. 1997; D'Amico et al. 2003; Errera 2004; Errera et al. 2004; D’Amico, 2005; Petrequin et al. 2005; 2008; 2011b; Thirault, 2005; Compagnoni et al. 2007).
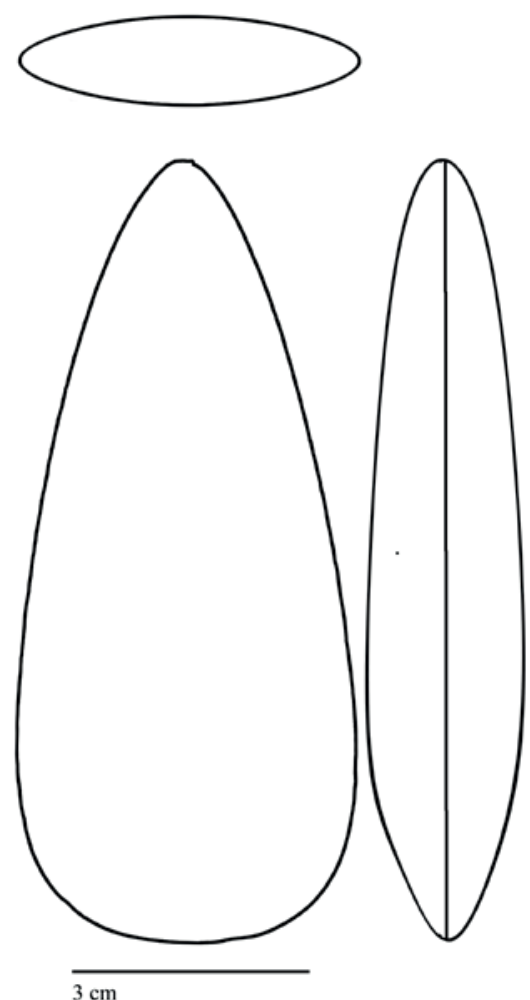

\section{ANÁLISIS}

\subsection{Concepto y definición de jade}

Jade, jadeitita o jadeíta son varios de los términos utilizados en la literatura arqueológica para definir de forma genérica a todas las rocas duras de color verde con las que se realizan útiles pulimentados (principalmente hachas, aunque también azuelas, cinceles, etc. Concretamente, 'jade' es un término ambiguo acuñado por Damour (1881) para describir comercialmente una gema y arqueológicamente el utillaje lítico verde pulimentado usado en la Prehistoria. El término jade se utiliza para designar dos tipos de mineralogías, jadeítas y actinolitas (nefritas), admitiéndose el uso de jade-jadeíta y jade-nefrita, este último ausente en el registro arqueológico de Europa Occidental durante la Prehistoria Reciente.

En Geología, jade-jadeita (Jd) es el nombre que recibe la especie mineral que responde a la composición química $\mathrm{NaAlSi}_{2} \mathrm{O}_{6}$. Se trata de uno de los miembros finales del grupo de los clinopiroxenos en el cual pueden pueden darse numerosas sustituciones químicas (Deer et al. 1992). Según Smith (2005a) las más frecuentes son las de: 
- $\mathrm{Na}^{+}$por $\mathrm{Li}^{+}, \mathrm{Ca}^{2+}, \mathrm{Mg}^{2+}, \mathrm{Mn}^{2+}, \mathrm{Fe}^{2+}$

- $\mathrm{AI}^{3+}$ por $\mathrm{Mg}^{2+}, \mathrm{Mn}^{2+}, \mathrm{Fe}^{2+}, \mathrm{Zn}^{2+}, \mathrm{Fe}^{+}, \mathrm{Cr}^{3+}, \mathrm{Mn}^{3+}$, $\mathrm{Sc}^{3+}, \mathrm{V}^{3+}, \mathrm{Ti}^{4+}$

- $\mathbf{S i}^{4+}$ por $\mathrm{AI}^{3+}, \mathrm{Fe}^{3+}, \mathrm{Ti}^{4+}$

El jade es una solución sólida, es decir, una mezcla de especies químicas en un fluido como en el caso de los inosilicatos del grupo piroxeno $\mathrm{XYZ}_{2} \mathrm{O}_{6}$ que incluyen las especies diópsido $\left(\mathrm{Ca}^{2+} \mathrm{Mg}^{2+} \mathrm{Si}_{2} \mathrm{O}_{6}\right)$ y hedenbergita $\left(\mathrm{CaFe}^{2+} \mathrm{Si}_{2} \mathrm{O}_{6}\right)$, donde la posición $\mathrm{X}$ es ocupada siempre por $\mathrm{Ca}$, y la posición $\mathrm{Y}$ puede ser ocupada por $\mathrm{Mg}$ como el diópsido puro, o por $\mathrm{Fe}^{2+}$ como en las hedenbergitas puras, y algunas veces por $\mathrm{Mg}$ y otras por $\mathrm{Fe}^{2+}$ como en la solución sólida diópsido-hedenbergita (Di-Hd). La composición media real del mineral viene dada por el promedio en la composición de $\mathrm{Mg}^{2+}$ y $\mathrm{Fe}^{2+}$ de la posición Y del volumen analizado (Smith 2005a). Existe, por tanto, una composición química que va de $\mathrm{Di}_{100} \mathrm{Hd}_{0}$ a $\mathrm{Di}_{0} \mathrm{Hd}_{100}$. En el caso que nos ocupa, el sistema diópsido $\left(\mathrm{Ca}^{2+} \mathrm{Mg}^{2+} \mathrm{Si}_{2} \mathrm{O}_{6}\right)$ - jadeíta $\left(\mathrm{Na}^{+} \mathrm{Al}^{3+} \mathrm{Si}_{2} \mathrm{O}_{6}\right)$, ambas posiciones pueden estar sujetas a mezclas, de tal forma que dos cationes heterovalentes se intercambian en vez de un único catión homovalente en la solución Di-Hd (Smith 2005a).

En la simetría C2/c de la jadeíta tan sólo existe una posición $\mathrm{X}$ y otra $\mathrm{Y}$, de tal forma que cuando se dan diferentes cationes en el cristal se distribuyen aleatoriamente. En la posición $\mathrm{Z}$ el catión $\mathrm{Si}^{4+}$ es reemplazado a un ratio relativamente bajo de presión/temperatura, y dado que la jadeíta sólo puede existir a altos ratios $\mathrm{P} / \mathrm{T}$, se pueden ignorar las sustituciones de $\mathrm{Si}^{4+}$ en esta solución sólida (Smith 2005a). Con el catión $\mathrm{Na}^{+}$fijo, las sustituciones más frecuentes son las de $\mathrm{Fe}^{3+}$ para formar aegirina (Ae, $\mathrm{NaFeSi}_{2} \mathrm{O}_{6}$ ) o las de $\mathrm{Cr}^{3+}$ para formar koshmochloro ( $\mathrm{Ks}, \mathrm{NaCrSi}_{2} \mathrm{O}_{6}$ ). La sustitución de cationes monovalentes de $\mathrm{Na}^{+}$por cationes divalentes hace que necesariamente se reemplacen los cationes trivalentes de $\mathrm{Al}^{3+}$ por cationes divalentes. Las sustituciones mejor conocidas son las de $\mathrm{Na}^{+} \mathrm{Al}^{3+}$ por $\mathrm{Ca}^{2+} \mathrm{Mg}^{2+}$ para formar diópsido ( $\mathrm{Di}, \mathrm{CaMgSi}_{2} \mathrm{O}_{6}$ ). Las sustituciones por $\mathrm{Fe}^{2+} \mathrm{o} \mathrm{Mn}^{2+}$ en vez de $\mathrm{Mg}^{2+}$ forman, respectivamente, hedenbergita $\left(\mathrm{Hd}, \mathrm{CaMgSi}_{2} \mathrm{O}_{6}\right)$ o johannsenita (Jo, CaMnSi ${ }_{2} \mathrm{O}_{6}$ ) (Smith 2005a; D’Amico et al. 2003).

Estos seis miembros finales (Ae, Ks, Di, Hd, Jo, Jd) son los más relevantes para la jadeíta, ya que en la naturaleza se suelen encontrar pequeñas cantidades de impurezas de $\mathrm{Ca}^{2+}, \mathrm{Mg}^{2+}, \mathrm{Mn}^{2+}, \mathrm{Cr}^{3+}$ y $\mathrm{Fe}^{3+} . \mathrm{Mg}^{2+}$, $\mathrm{Fe}^{2+} \mathrm{O} \mathrm{Mn}^{2+}$ que ocupan el lugar del $\mathrm{Ca}^{2+}$ en la posición Y. Estas soluciones sólidas pueden ocurrir en el grupo de los piroxenos, especialmente en el sistema DiJd, entre la jadeíta y el diópsido. Entre estos miembros finales existen no miles, sino millones de composiciones químicas diferentes para un mismo grupo mineral. Entre ellas podemos encontrar la onfacita que se corresponde con la composición intermedia $\mathrm{Di}_{50} \mathrm{Jd}_{50}$ $\left(\mathrm{Na}_{0.5} \mathrm{Ca}_{0.5} \mathrm{Mg}_{0.5} \mathrm{Al}_{0.5} \mathrm{Si}_{2} \mathrm{O}_{6}\right)$ y es el constituyente típico de las eclogitas (D’Amico et al. 1995; 2003; Gendron et al. 2002; Smith 2005a).

Partiendo de los trabajos de Ricq-de-Bouard (Ricqde-Bouard et al. 1990; 1993; Ricq-de-Bouard y Fedele, 1993) tanto Petrequin et al. (1997, 2005, 2011b) como D'Amico et al. $(1995,2003)$ definen tres grandes grupos de rocas asociadas al termino jade y utilizadas en la elaboración de utillaje lítico pulimentado durante la Prehistoria Reciente: los jade-jadeítas, las onfacitas y las eclogitas. Tanto las jadeítas, las onfacitas como las eclogitas se definen por ser una solución sólida de piroxenos en el sistema Di-Jd. Utilizan por tanto, el término jade-jadeíta como un sinónimo de piroxeno-Na puro y onfacita (-jadeíta) es una solución sólida de jadeíta-diopsido (Deer et al. 1992) que se utiliza para definir litologías similares a las eclogitas en las que el granate está ausente (D’Amico et al. 1995; 2003; Ricq-de-Bouard et al. 1990; Ricq-de-Bouard y Fedele, 1993). La gran diferencia entre onfacitas y eclogitas es precisamente la presencia de granate en proporciones superiores al 5\% en las eclogitas (D'Amico et al. 1995; 2003; Ricq-de-Bouard et al. 1990; Ricq-de-Bouard y Fedele, 1993).

\subsection{Metodología}

La petrografía es la técnica más extendida en el estudio de las hachas alpinas, pero también la más agresiva y destructiva. El uso de técnicas de análisis modernas y no destructivas como la difracción de rayos X (XRD), micro sondas de fluorescencia de rayos $X(\mu-X R F)$, espectrometría difusa confocal micro-raman (DC $\mu \mathrm{RS})$ o la radio-espectrometría UV-VIS-NIR han resultado ser extremadamente útiles a la hora de comparar las fuentes con los productos particularmente en el caso de las jadeítas. Estas técnicas permiten establecer diferencias a nivel mineralógico y composicional, una información que ayuda notablemente a tener una visión mucho más amplia de las pautas de intercambio y circulación del jade-jadeíta en la Europa prehistórica.

La difracción de rayos $\mathrm{X}$ es una técnica barata $\mathrm{y}$ de gran resolución que informa acerca de la composición mineralógica, cuantificando groseramente la proporción de piroxenos en la mezcla heterogénea de una solución sólida (Chiari et al. 1996a; D’Amico et al. 1995; 2003; 
Giustetto et al. 2008). La difracción de rayos X es utilizada para identificar los minerales que componen la pieza. Tras el cálculo del fondo con el software X'Pert Highscore Plus 3.0 y la identificación de todos los picos existentes en el diagrama, los valores numéricos obtenidos se han comparado con la base de datos ICDD (International Centre for Difraction Data) PDF (Powder Difraction File) 2004 con el objetivo de identificar los minerales que componen la muestra.

La espectroscopia $\mu$-raman, técnica igualmente no destructiva, sirve para identificar/caracterizar sólidos inorgánicos, especialmente cuando se trabaja a escala micrométrica con mezclas heterogéneas a través de las vibraciones de la red cristalina, siendo sensible a la composición, los enlaces, el entorno de coordinación y la estructura cristalina de la muestra (Smith y Clark, 2004). Uno de los aspectos más importantes que se pueden investigar utilizando esta técnica es la clasificación de las rocas en términos de especie mineral, pureza y procedencia. El uso de la DC $\mu$ RS es una técnica bien conocida para el estudio de gemas y minerales, cuya ventaja consiste en ser no invasiva y no destructiva.

Recientemente Errera (2004) utilizando espectroradiometría de reflectancia difusa UV-VIS-NIR, ha clasificado las hachas en función a su mineralogía. Aunque según apuntan otros autores (Giustetto et al. 2008) estos resultado pueden ser dudosos dado que la asociación/mezcla de diferentes mineralogías puede dar resultados muy similares.

A pesar de que el color es uno de los atributos más importantes de estas hachas y que recientemente se ha hecho una clasificación basada en este parámetro (Petrequin et al. 2011b), no parece haber ninguna mención objetiva y precisa en la literatura al uso a este respecto. El color es siempre referido como una apreciación visual en términos de oscuro, claro, brillante, etc. terminología a todas luces subjetiva e imprecisa. Según la teoría triestímulo del color, éste se define por tres características básicas que son observables científicamente de una forma objetiva: el tono, la claridad y la saturación. Estos tres valores pueden ser medidos según la CIE (1976) en el espacio de color unificado por las coordenadas de color CieLa*b* o CieLC ${ }_{\mathrm{a}, \mathrm{b}} \mathrm{h}_{\mathrm{a}, \mathrm{b}}$ donde $\mathrm{C}_{\mathrm{a}, \mathrm{b}}^{*}$ (chroma) representa la mezcla de los componentes cromáticos y no cromáticos del tono o la pureza del tono, $\mathrm{h}_{\mathrm{a}, \mathrm{b}}$ (hue) el tono y L (lightness) la claridad. Por tanto a nivel colorimétrico y para evitar una apreciación subjetiva del color hemos realizado una medida del color a través de fotografías calibradas utilizando un iluminante D50 y el espacio unificado de color CieLa*b* (1976) y el observador estándar.
A nivel composicional se ha utilizado un equipo de EDAX Eagle III de $\mu$-FRX. Tras colocar la pieza en una cámara en vacio, ésta se enfoca con un microscopio óptico. Se analizó un área de $100 \mu \mathrm{m}$ de la pieza usando la radiación $\mathrm{K} \alpha$ producida por un tubo de $\mathrm{Rh}$ operado a $40 \mathrm{kV}$. La cuantificación se ha realizado usando el paquete informático del equipo que no requiere patrones. Los resultados cuantitativos se presentan como el promedio de cinco medidas.

A nivel mineralógico se ha optado por utilizar un equipo de difracción de rayos $\mathrm{X} \theta / \theta$ Panalytical X'Pert Pro con radiación $\mathrm{Cu} \mathrm{K} \alpha(1.5406 \AA$ Å) operado a $45 \mathrm{kV}$ y $40 \mathrm{~mA}$ equipado con un detector PixCel y espejos parabólicos de haz incidente. Los diagramas se adquieren con un paso de $0,026^{\circ} 2 \theta$ entre $10^{\circ}$ y $70^{\circ}$ $2 \theta$ con un tiempo de adquisición de 247 s por paso a temperatura ambiente $\left(25^{\circ} \mathrm{C}\right)$. En la fig. 2 a podemos ver un fotomontaje de la fluorescencia producida por el haz de rayos $\mathrm{X}$ al incidir sobre una película fluorescente, el área de barrido y la zona de análisis en la muestra. El área barrida es de c 2 x 0,5 cm, área más que representativa de la mineralogía presente en este sólido cristalino heterogéneo.

Estos datos han sido completados utilizando un espectrómetro dispersivo $\mu$-raman confocal (DC $\mu \mathrm{RS}$ ) HORIBA Jobin Yvon LabRAM HR system. El diodo laser operado a una longitud de onda $532,06 \mathrm{~nm}$ produce una potencia de hasta $15 \mathrm{~mW}$ en la fuente. No se utilizaron filtros para reducir la potencia del láser. El tiempo de adquisición fue de $32 \mathrm{~s}$ por adquisición hasta un máximo de 20. El rango espectral de medida elegido fue entre 100 y $1800 \mathrm{~cm}^{-1}$ utilizando un objetivo $100 \mathrm{x}$ con detector CCD multicanal. La precisión de la medida seleccionada es de $1 \mathrm{~cm}^{-1}$. El área de medida seleccionada fue de $1000 \mathrm{~mm}$ de diámetro (fig. 2b).

\subsection{Resultados}

En la figura 3 se aprecia el diagrama obtenido para el hacha de la Colección Tubino (VAL001) comparado con los diagramas de rayos $\mathrm{X}$ obtenidos por D'Amico et al. $(2003 ; 2005)$ de rocas meta-ofiolíticas utilizadas en la producción de hachas alpinas y las fichas de la base de datos ICDD PDF correspondientes a los piroxenos-Na jadeíta (01-071-1506) y onfacita (01-070-1874) pertenecientes al sistema Di-Jd. Podemos además observar que en la muestra no hay trazas de granate (Grt en la fig. 3) lo que anula la posibilidad de que la muestra pueda considerarse una eclogita (D'Amico et al. 2003). Tal y como puede apreciarse por la coincidencia 

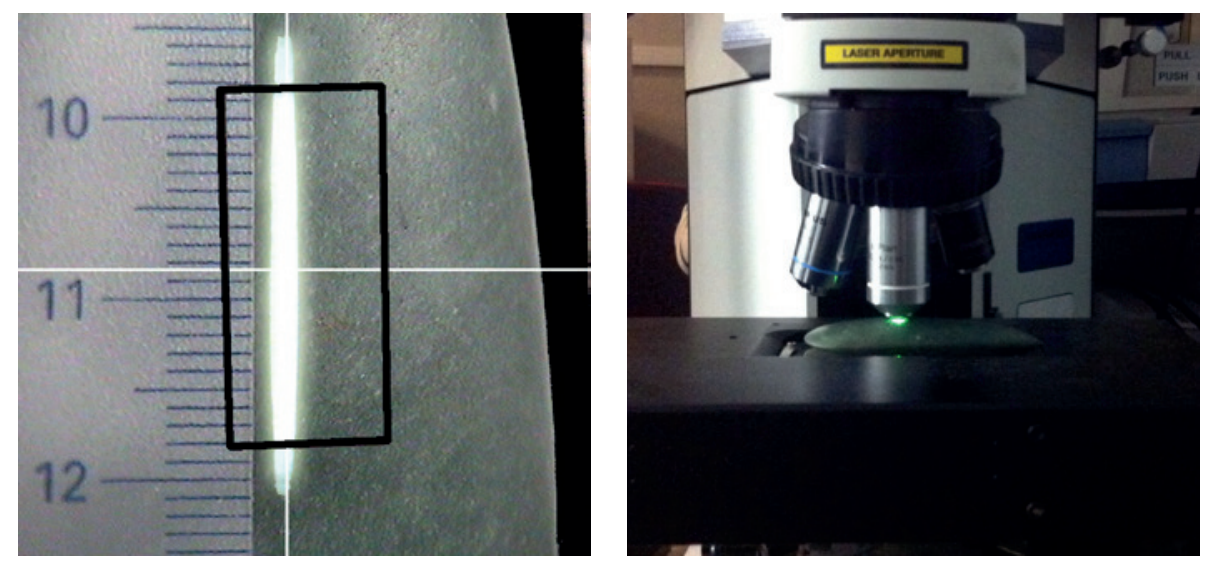

Figura 2: a. fotomontaje del área analizada por XRD de la muestra; $b$. análisis no destructivo mediante $\mathrm{DC} \mu \mathrm{RS}$.

los planos [hkl 221 y 310], estamos tratando con una jadeíta que puede presentar trazas de onfacita, según se desprende de los hombros asociados a dichas difracciones. El diagrama de rayos X de la muestra VAL001 y el diagrama de la jadeíta (01-071-1506) son plenamente coincidentes, aunque podemos apreciar en la muestra VAL001 un pequeño hombro en el pico correspondiente al plano [hkl 221] que coincide con la difracción principal [hkl 221] de la onfacita (01-070-1874). La muestra, por tanto, podría considerarse como una solución sólida heterogénea de jadeíta y onfacita. En cualquier caso, a tenor de la intensidad de dicho hombro, la proporción de onfacita sería muy escasa y en ningún caso podría considerarse un jade mixto, ya que este presenta dos dobletes de picos en el intervalo 30-32 ${ }^{\circ} 2 \theta$. Esto puede apreciarse con claridad en la figura 3, ya que el perfil de la muestra VAL001 coincide con el de jadeíta de D'Amico et al. (2003) y no con el jade mixto (Chiari et al. 1996b; D’Amico et al. 2003).

Los planos principales de los piroxenos-Na: jadeíta y onfacita, se encuentran en el intervalo 30-32 ${ }^{\circ} 2 \theta$, donde se ubican los planos con las difracciones más intensas de la serie Di-Jd. Las reflexiones que por ser las más intensas, estar próximas y bien separadas presentan mejores características para la identificación de fases son la 221 y la 310 (D'Amico et al. 1995; 2003; Chiari et al. 1996b; Giustetto et al. 2008). Ambos planos [hkl 221 y 310$]$ presentan variaciones significativas en toda la serie de soluciones sólidas pasando de 30,70$31,60^{\circ} 2 \theta$ (jadeíta) a $29,84-30,27^{\circ} 2 \theta(\mathrm{Wo}+\mathrm{En}+\mathrm{FS})$ respectivamente con valores intermedios para la Aegirina (Giustetto et al. 2008). Es, por tanto, esta región la que se utiliza para identificar la presencia de jadeíta/onfacita (D’Amico et al. 1995; 2003; Giustetto et al. 2008). Las fases que se identifican por norma general no son fases puras y la cristalización, por tanto, suele ser una mezcla heterogénea de distintos cristales dentro de la serie, por lo que es posible que existan desplazamientos de las difracciones dentro los rangos anteriormente citados, a lo que hay que añadirle un posible desplazamiento debido a que la superficie de medida no es perfectamente plana, y a pesar de que el uso de espejos parabólicos unidos al haz paralelo evitan los problemas de textura de la muestra, no corrigen el desplazamiento o la mala resolución de los picos derivado de una muestra que nos es completamente plana. A pesar de que otros autores (Chiari et al. 1996b) opinan que con esta técnica las posiciones angulares y las intensidades son muy precisas en muestras planas, aunque no tanto las intensidades en muestras curvas, nosotros encontramos que los picos quedan mal definidos o resueltos, siendo anchos y con hombros.

Observando este intervalo, 30-32 ${ }^{\circ} 2 \theta$ (fig. 4), se puede apreciar cómo las difracciones registradas suponen dos picos anchos con hombros poco o mal resueltos en los planos [hkl 221 y 310] correspondientes a la jadeíta. Para resolver estos picos se han deconvolucionado, lo que ha resultado en la obtención de cuatro curvas, que se corresponden con los planos [hkl 221: $30,43^{\circ} 2 \theta$ y $310: 31,48^{\circ} 2 \theta$ ] de la jadeíta. Las otras dos difracciones a $29,99^{\circ} 2 \theta$ y $31,14^{\circ} 2 \theta$ no encajan con ninguna de las fichas presentes en la base de datos ICDD PDF para la jadeíta o la onfacita, sin embargo, la difracción a $29,99^{\circ} 2 \theta$ y a $31,14^{\circ} 2 \theta$ coincide con los plano [hkl -221 y 130] de la ficha 01-080-186 para los clinopiroxenos (Al 0,32 $\mathrm{Ca} 0,55 \mathrm{Fe} 0,15 \mathrm{Mg} 0,65 \mathrm{Na} \mathrm{0,3} \mathrm{O} 6$ Si 2 Ti 0,01) con espaciado C2/c en el sistema Di-HdJd. Por tanto, ante la ausencia de otros minerales minoritarios o traza, es posible pensar que estas dos líneas se corresponden con alguna fase dentro de la serie. La jadeíta es un miembro final del grupo de los piroxenos en el que pueden darse numerosas sustituciones químicas. Estas sustituciones serían las responsables de las dos 




difracciones que harían oscilar las difracciones principales de la jadeíta en los intervalos 30,70-31,60² $\theta$.

Los resultados del análisis composicional que se presenta en la tabla 1 vendrían a confirmar que la pieza en cuestión es una jadeíta que presenta sustituciones de $\mathrm{Ca}^{2+} \mathrm{y} \mathrm{Fe}^{2+}$ en proporciones relativamente bajas, es decir una jadeíta con un bajo grado de impurezas dentro del sistema Di-Hd-Jd.

La espectroscopia DC $\mathrm{DRS}$ es una técnica idónea para la clasificación de las rocas en términos de especie mineral, pero sobre todo de pureza y procedencia que ha sido escasamente aplicada a los jades europeos y algo más profusamente a los jades Maya (Smith y Gendron 1997; Smith y Clark 2004; Smith 2005a; 2005b; Edwards y Chalmers 2005). Las bandas vibracionales entre 1800-100 $\mathrm{cm}^{-1}$ registradas en el espectro DC $\mu \mathrm{RS}$ del hacha de la colección Tubino se corresponden con las que presenta la jadeíta en la ficha 565 de la base de datos HORIBA Jobin Yvon KnowItAll ${ }^{\circledR}$ para materiales inorgánicos, minerales y gemas (fig. 5).

Una característica de los espectros $\mu$-raman de jadejadeíta es el predominio de las bandas correspondientes a la vibración simétrica Si-O-Si de los modos de tensión del enlace oxígeno en los inosilicatos que ocurren en el intervalo 650-750 $\mathrm{cm}^{-1}$ (Smith 2005a; Smith y Gendron 1997). En el espectro se han identificado 14 bandas en el intervalo $1200-150 \mathrm{~cm}^{-1}$. Entre ellas se identifica fácilmente la intensa banda simétrica $\mathrm{Si}-\mathrm{O}-\mathrm{Si}$ de los modos de tensión a $702 \mathrm{~cm}^{-1} \mathrm{y}$ una banda fuerte a $1044 \mathrm{~cm}^{-1}$ acompañada de una banda más débil y ancha 


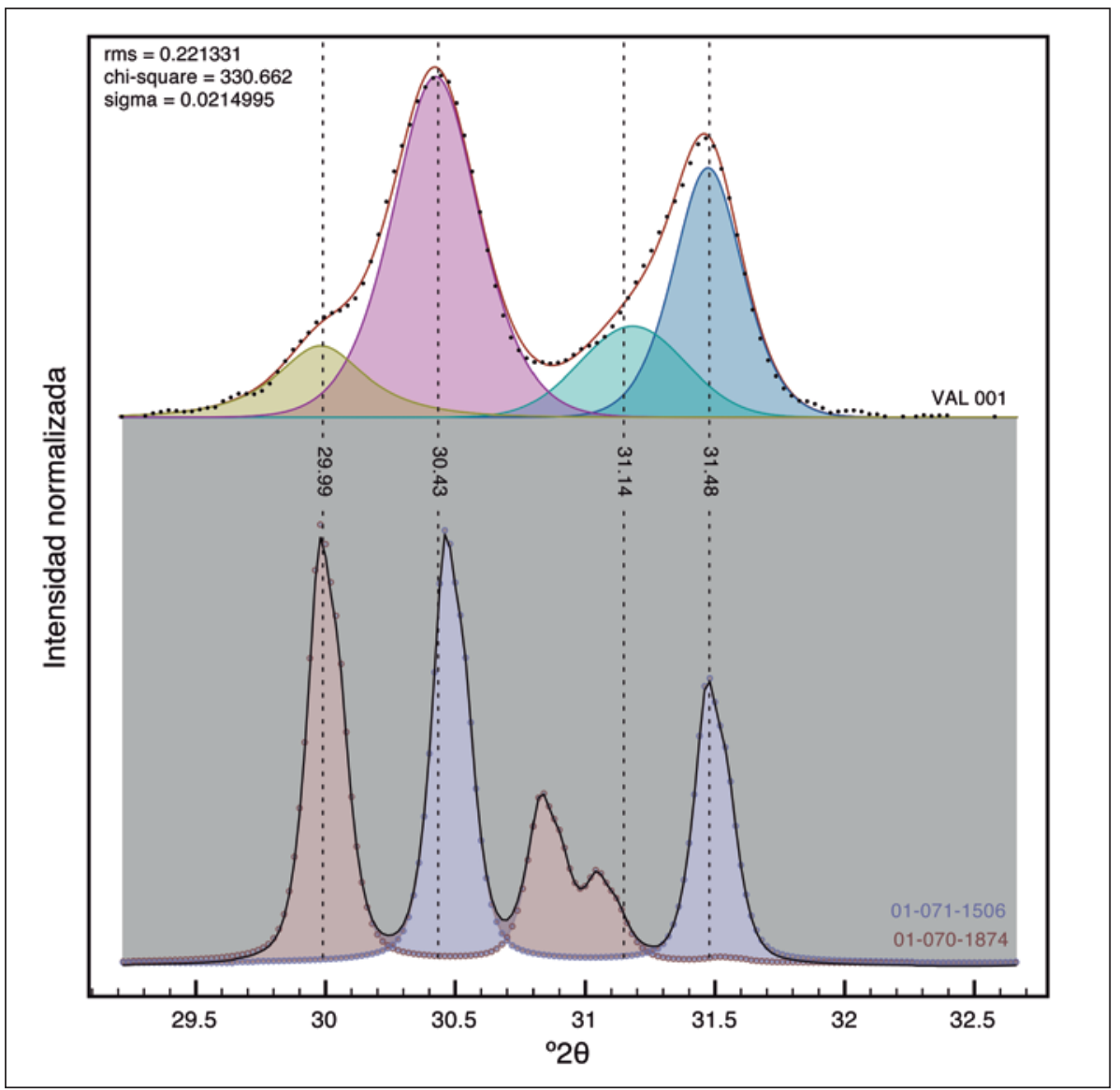

Figura 4: Deconvolución de los picos observados en el intervalo $30.70-$ $31.60^{\circ} 2 \theta$ del XRD.

a $994 \mathrm{~cm}^{-1}$, que se han atribuido a las vibraciones del tetraedro $\mathrm{SiO}_{4}$ (Smith 2005a). Otras dos bandas fuertes ocurren a 373 y $203 \mathrm{~cm}^{-1}$, que junto a las restantes 9 bandas conforman el espectro raman característico del miembro final de los piroxenos-Na, jadeíta. Las tres líneas más fuertes presentan valores ligeramente superiores que los del hacha de jade-jadeíta (no. 103.434) de Falcon (Francia), descrita por Damour (1865) y analizada mediante microscopia raman por Smith (2005a). De hecho estos valores ligeramente superiores indica que tratamos con una composición ligeramente más rica en jadeíta que el hacha analizada por Smith (2005a) cuyo $\mathrm{mol} \% \mathrm{Jd}=90 \mu$ 0,5\%. La banda simétrica Si-O-Si de los modos de tensión c. $700 \mathrm{~cm}^{-1}$ es, de hecho, un gran indicador cuantitativo de la pureza de un piroxeno, ya que en los otros miembros finales comunes como el diópsido, la hedenbergita y la aegirina ocurre a longitudes de onda mucho menores (Smith 2005a; Smith y Gendron 1997). En este sentido, los modos de tensión Si-O-Si para el diópsido y la hedenbergita ocurren a 666 y $660 \mathrm{~cm}^{-1}$ respectivamente, de manera muy similar y a la vez diferente a como ocurre en la jadeíta: $703 \mathrm{~cm}^{-1}$ (Smith 2005a). Si asumimos, tal y como indica Smith (2005a), que la longitud de onda varia linealmente con la composición química desplazándose como consecuencia de los cambios en la masa atómica, radio iónico y/o carga electrónica de los elementos químicos - diferentes proporciones de $\mathrm{Ca}, \mathrm{Mg}$, $\mathrm{Na}$ o Al en el sistema Di-Hd-Jd (Smith 2005b)-, y que no se producen sustituciones químicas significativas, tal y como hemos podido observar en la tabla 1, entonces el porcentaje molar $(\% \mathrm{Jd})$ en el sistema Di-Hd-Jd viene dado directamente por la longitud de onda cambiante de la banda simple Si-O-Si. Y dado que la mayoría de las jadeítas naturales contienen esencialmente Di-Hd-Jd como miembros finales, el desplazamiento de la banda simple Si-O-Si en el espectro raman determinará el porcentaje aproximado de jadeíta (Smith 2005a). De tal manera que siempre que esta banda aparezca a longitudes de onda superiores a $700 \mathrm{~cm}^{-1}$ la proporción relativa de jadeíta será de al menos el $95 \mathrm{~mol} \%$ (Smith 2005b). 
Figura 5: Espectro

DC $\mu$ RS comparado de la muestra VAL001 y el espectro de una jadeíta (ficha 565).

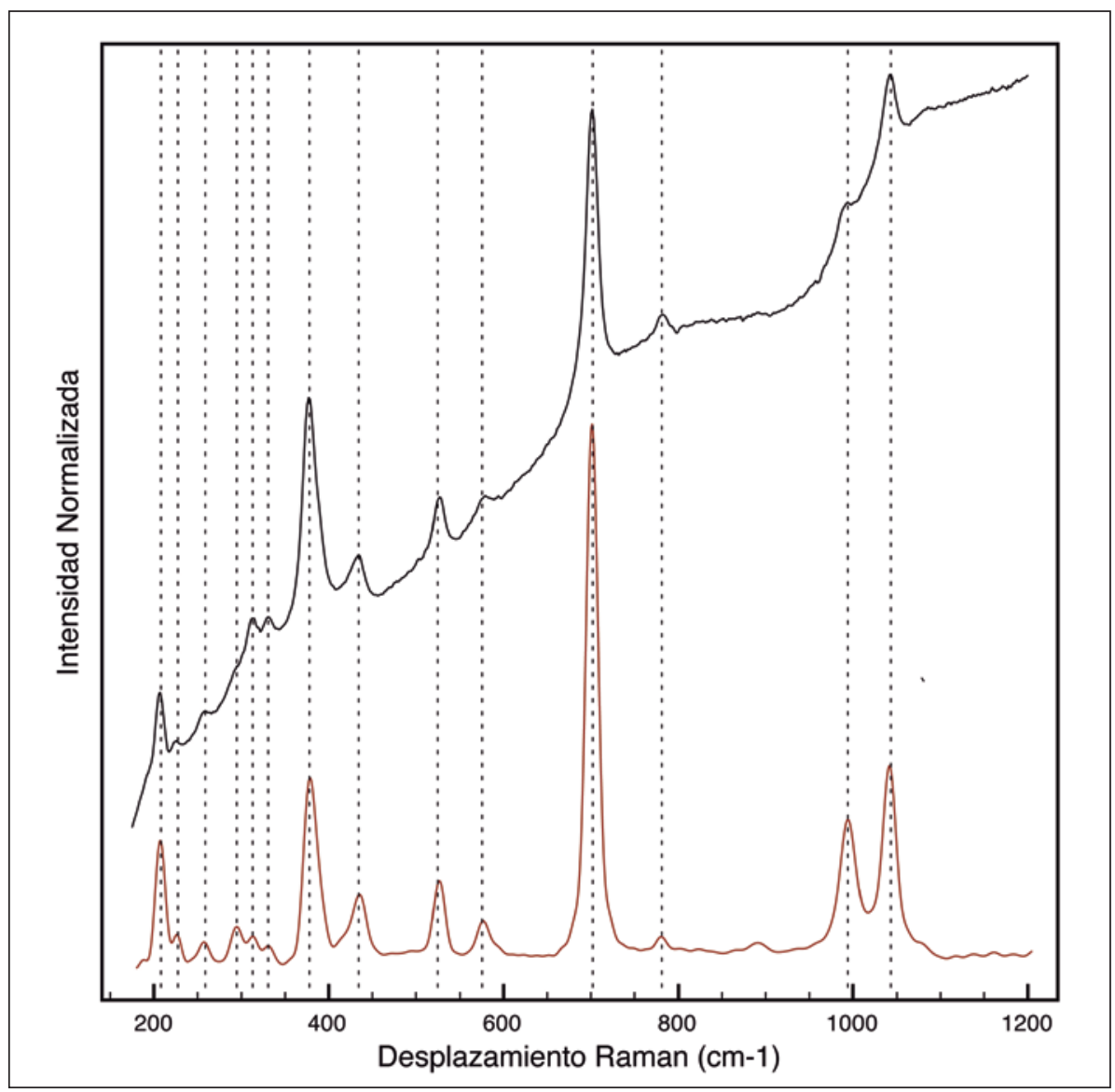

Tabla 1: resultados composicionales obtenidos mediante $\mu$-FRX. Resaltados en gris las impurezas a nivel de trazas.

\begin{tabular}{|c|c|c|c|c|c|c|}
\hline Elem. & Net & Wt $\%$ & At $\%$ & I-Error $\%$ & BG & Wt-Error \\
\hline $\mathrm{Na}$ & 14.05 & 7.37 & 9.09 & 3.90 & 7.98 & 0.31 \\
\hline $\mathrm{Al}$ & 260.78 & 20.34 & 21.37 & 0.64 & 8.42 & 0.35 \\
\hline $\mathrm{Si}$ & 1221.51 & 63.48 & 64.08 & 0.29 & 7.00 & 1.03 \\
\hline $\mathrm{S}$ & 10.56 & 0.32 & 0.29 & 4.37 & 5.35 & 0.02 \\
\hline $\mathrm{K}$ & 15.91 & 0.54 & 0.39 & 3.45 & 7.14 & 0.02 \\
\hline $\mathrm{Ca}$ & 160.43 & 3.76 & 2.66 & 0.83 & 7.44 & 0.07 \\
\hline $\mathrm{Ti}$ & 11.40 & 0.14 & 0.08 & 4.63 & 8.22 & 0.01 \\
\hline $\mathrm{V}$ & 8.78 & 0.08 & 0.05 & 5.97 & 9.35 & 0.01 \\
\hline $\mathrm{Mn}$ & 24.72 & 0.16 & 0.08 & 2.98 & 14.71 & 0.01 \\
\hline $\mathrm{Fe}$ & 682.58 & 3.71 & 1.88 & 0.39 & 17.48 & 0.06 \\
\hline $\mathrm{Cu}$ & 12.04 & 0.05 & 0.02 & 6.11 & 21.06 & 0 \\
\hline $\mathrm{Zr}$ & 9.86 & 0.03 & 0.01 & 8.35 & 28.95 & 0 \\
\hline
\end{tabular}


Tabla 2: valores tabulados para la posición de las bandas obtenidas mediante DC $\mu$ RS y su identificación de los modos vibracionales asociados de la muestra VAL001 y del hacha pulimentada de Falcon (Francia) no. 103.434

(Smith 2005a).

\begin{tabular}{|c|c|c|}
\hline $\begin{array}{c}\text { Damour } \\
\left(\mathrm{cm}^{-1}\right)\end{array}$ & $\begin{array}{l}\text { VAL001 } \\
\left(\mathrm{cm}^{-1}\right)\end{array}$ & Causas \\
\hline 204 & 208 & Libracional y rotacional (Hatipofülu et al., 2012) \\
\hline 221 & 225 & Libracional y rotacional (Hatipofülu et al., 2012) \\
\hline 254 & 258 & Libracional y rotacional (Hatipofülu et al., 2012) \\
\hline 290 & 294 & Libracional y rotacional (Hatipofülu et al., 2012) \\
\hline 309 & 313 & Libracional y rotacional (Hatipofülu et al., 2012) \\
\hline 326 & 330 & $\begin{array}{l}\text { Modo } v_{2} \text { de flexión simétrico simple de }\left(\mathrm{SiO}_{4} / \mathrm{M}\right) \text { (Hatipofülu et al., 2012); } \\
\text { Modo de tensión de Na-O (Huang, 1999) }\end{array}$ \\
\hline 375 & 377 & Modo vibracional de Al-O (Huang, 1999) \\
\hline \multicolumn{3}{|l|}{386} \\
\hline 430 & 434 & Modo vibracional Al-O (Huang, 1999) \\
\hline 525 & 527 & Modo de flexión Si-O (Huang, 1999) \\
\hline 574 & 576 & $\begin{array}{l}\text { Modo } v_{2} \text { de flexión simétrica del cuarto centro degenerado de }\left(\mathrm{SiO}_{4} / \mathrm{M}\right) \\
\text { (Hatipofülu et al., 2012) }\end{array}$ \\
\hline 699 & 702 & $\begin{array}{l}\text { Modo de tensión simétrico de Si-O-Si (Tuncer Arslanlar et al., 2011, } \\
\text { Smith and Gendron, 1997) }\end{array}$ \\
\hline 778 & 782 & \\
\hline 991 & 994 & Modo de tensión simétrico de Si-O-Si (Tuncer Arslanlar et al., 2011) \\
\hline 1040 & 1044 & $\begin{array}{l}\text { Vibraciones internas del tetraedro } \mathrm{SiO}_{4}\left(\mathrm{Smith}_{\text {and }} \text { Gendron, 1997), modo } v_{1} \text { de tensión }\right. \\
\text { doble simétrica de }\left(\mathrm{SiO}_{4} / \mathrm{M}\right)(\text { Hatipofülu et al., 2012) }\end{array}$ \\
\hline
\end{tabular}

* Hacha pulimentada Neolítica de jadeíta (no. 103.434) de Falcon (Francia), descrita por Damour (1865), $90 \pm 0.5$ mol.\% jadeíta (datos de Smith, 2005a).

El hacha de la Colección Tubino presenta la banda simple Si-O-Si a $702 \mathrm{~cm}^{-1}$ (tabla 2), lo que implicaría que, siguiendo a Smith (2005a), el \%Jd $=97.7 \%$. La muestra, por tanto, se compone mayoritariamente de clinopiroxeno con un contenido de jadeita $>$ al $97 \mathrm{~mol} \%$, pudiéndose considerar casi un mono-mineral, que coincide certeramente con la definición arqueológica y gemológica de jadeíta (jade). La espectrometría $\mu$-raman confirma de esta manera los datos obtenidos mediante $\mathrm{XRD}$, que tan sólo existe una fase mineral dominante en la muestra, la jadeíta. Por tanto, esta pieza no es una solución sólida en el sistema Di-Hd-Jd, sino una jadeíta impura donde se dan sustituciones minoritarias.

Un último aspecto analizado es del color de la pieza. A pesar de que el color es uno de los atributos más importantes de estas hachas, pues parece determinar su distribución, uso y valor simbólico (Cooney 2002), no hay en la literatura ningún análisis científico del mismo en términos de los tres componentes del color (tono, claridad y pureza), algo especialmente importante a la luz de que el lenguaje determina en cierta medida cómo definimos los colores (Hardin y Maffoi 1997; Jones y MacGregor 2002). La medida precisa y certera del color de las piezas cobra aún más importancia si tenemos en cuenta que las sociedades de la Prehistoria Reciente no catalogaban las rocas en función de sus características mineralógicas sino de sus características visuales, es decir, de la percepción que de ellas se tenía. El color (fig. 6) del hacha de la Colección Tubino es de tono verde $\left(\mathrm{h}_{a, \mathrm{~b}}=161^{\circ}\right)$ relativamente claro $(\mathrm{L}=37.75)$ y bastante saturado $\left(\mathrm{C}^{*}{ }_{\mathrm{a}, \mathrm{b}}\right.$ $=12.67$ ). A pesar de las variaciones en $h_{a, b}$ que se han medido (tabla 3) el tono es relativamente uniforme y su variación se ubica dentro del umbral de variación que no es perceptible visualmente $\left(\mathrm{h}_{\mathrm{a}, \mathrm{b}} \pm 3^{\circ}\right)$. 
Figura 6: coordenadas de color en el UCS CieL*a*b* donde cada punto representa el valor promedio de un área de 101x101 pixeles. Los colores representados en la gráfica no son más que una decoración, son los valores de $\mathrm{a}^{*} \mathrm{y} \mathrm{b}^{*}$ los que determinan el tono del hacha.

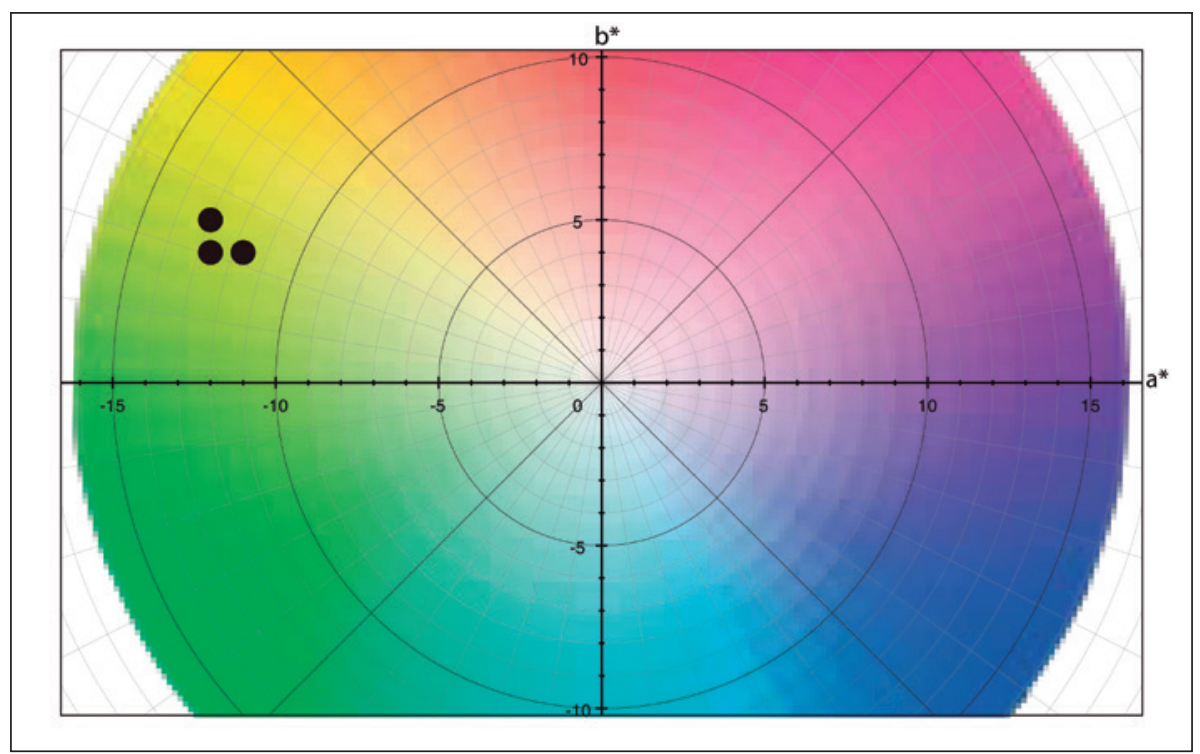

Tabla 3: coordenadas de color en el UCS CieL*a*b* y CieLCh donde cada punto representa el valor promedio de un área de 101x101 pixeles.

\begin{tabular}{|c|c|c|c|c|c|}
\hline $\mathrm{L}^{*}$ & $\mathrm{a}^{*}$ & $\mathrm{~b}^{*}$ & $\mathrm{~L}$ & $\mathrm{C}_{\mathrm{a}, \mathrm{b}}^{*}$ & $\mathrm{~h}_{\mathrm{a}, \mathrm{b}}$ \\
\hline 38.00 & -12.00 & 4.00 & 38.00 & 12.65 & 161.57 \\
\hline 39.00 & -11.00 & 4.00 & 39.00 & 11.70 & 160.02 \\
\hline 32.00 & -12.00 & 5.00 & 32.00 & 13.00 & 157.38 \\
\hline
\end{tabular}

\section{DISCUSIÓN}

Una vez que, mediante la aplicación de dos técnicas analíticas diferentes (XRD, DC $\mu$ RS), el hacha de la Colección Tubino ha sido definida mineralógicamente como jade-jadeíta de una pureza superior al $95 \mathrm{ml} \%$, es posible afirmar que la pieza debe proceder de los Alpes, dado que las únicas fuentes de esta roca se encuentran en esa región. Aunque la mayoría de los/as especialistas en esta materia prima afirman que las únicas fuentes de jade conocidas para Europa están en los Alpes, es imposible determinar el origen exacto dentro de las múltiples fuentes alpinas localizadas, bien de los depósitos secundarios de materiales de arrastre de los glaciares o de los conglomerados terciarios del Piamonte alpino (Ricq-de-Bouard y Fedele 1993; D'Amico et al. 1995; 2003; Ricq-de-Bouard et al. 1990; Petrequin et al. 2006), bien de las zonas de explotación primaria de Oncino en Mont Viso (Errera 2004; Petrequin et al. 2006; Compagnoni et al. 2007). Ello es debido a que las fuentes primarias han sido localizadas recientemente y todavía falta un ingente trabajo de caracterización de las mismas, para lo cual el primer paso es dar con una técnica analítica que sea capaz de detectar diferencias entre las fuentes. Recientemente, y a este respecto, se ha propuesto el uso de la radioespectrometría VIS-UV (Errera et al. 2004) como una técnica prometedora a la hora de discriminar entre distintas fuentes, pero a la que aún le queda un largo camino por recorrer para ser plenamente fiable. A este respecto la espectrometría raman que no ha sido aplicada al estudio de las hachas ni de las fuentes alpinas, presenta una trayectoria consolidada. Con ella no sólo es posible identificar el mineral y su pureza, sino que en base a los desplazamientos raman es posible caracterizar certeramente las fuentes, ya que este desplazamiento depende en última instancia de las sustituciones en el sistema Di-Hd-Jd.

Aparte de en los Alpes, concretamente del Piamonte alpino, a tenor de los estudios petrológicos (Ricq-deBouard et al. 1990; D'Amico et al. 1995; D'Amico et al. 2003; D'Amico, 2005; Compagnoni et al. 2007; Ricq-de-Bouard, 1993; Ricq-de-Bouard y Fedele, 1993) y la radioespectrometría (Errera 2004; Petrequin et al. 2005; Errera et al. 2004), en los alrededores de Mont Viso y en el macizo de Mont Beigua en los Alpes Occidentales (Ricq-de-Bouard et al. 1990; Ricq-de-Bouard 1993; Ricq-de-Bouard y Fedele 1993; D'Amico et al. 1995; Petrequin y Jeunesse 1995; Petrequin et al. 1997; D'Amico et al., 2003, Errera, 2004, Errera et al., 2004, D'Amico, 2005, Petrequin et al., 2005, Thirault, 2005, 
Compagnoni et al., 2007, Petrequin et al., 2008, Petrequin et al., 2011b), en Europa Occidental tan sólo se conocen pequeños afloramientos de piroxenos-Na de tipo jadeíta en la Bretaña francesa (Pin y Vielzeuf 1988). En la Península Ibérica al parecer existe algún afloramiento de eclogitas en la Cordillera Bética $\left(\mathrm{Jd}_{44} \mathrm{Di}_{6}-\right.$ $\mathrm{Jd}_{35} \mathrm{Di}_{12}$ con granates y anfibolitas) (Morten et al. 1987) pero no de jadeítas.

Distintos trabajos (Petrequin et al. 2002; 2011b; Petrequin y Jeunesse 1995) han propuesto la existencia de una línea entre Ginebra y Le Havre, a 500 km de los Alpes, que separaría el área de distribución de eclogitas, verdes oscuras, de la de las jadeítas, verdes claras (Petrequin et al. 2011b). Esta división no es relativa a la percepción del color de las hachas sino también a su función y valores simbólicos ya que las hachas 'verde claro' realizadas en jadeíta, habrían tenido un valor simbólico, mientras que las hachas 'verde oscuro', realizadas en ofacitas/eclogitas, habrían tenido un valor puramente funcional. Ello viene atestiguado por la mayor inversión de trabajo en el pulido de las jadeítas (Risch 2011) y el contexto de aparición (Petrequin et al. 2011b). El color, la forma y la textura del hacha de la colección Tubino encajarían dentro de la estrategia de explotación y distribución de los diferentes tipos de rocas alpinas. En concreto con aquellas jadeítas que presentan colores claros y son translúcidas que predominan en la cuenca de París, Alemania, Gran Bretaña e Irlanda (Petrequin et al. 2011b)

En ciertas partes de Europa (sur de Francia e Italia) las grandes hachas realizadas en eclogitas/onfacitas oscuras son mayoritarias, mientras que en la cuenca de París, Bretaña, e Irlanda las eclogitas/onfacitas representan una minoría dentro del registro arqueológico donde predominan las hachas verdes claras y translúcidas de jadeíta (Petrequin et al. 2011b). Esta pauta tiene además una correlación tipológica y cronológica, ya que por norma general las hachas realizadas en eclogita/onfacita y distribuidas a menos de $500 \mathrm{~km}$ de los Alpes, al sur de la línea Ginebra-Le Havre (Petrequin et al. 2002; 2011b; Petrequin y Jeunesse 1995), son largas, finas, de sección gruesa y color verde oscuro, mientras que las realizadas en jadeíta y distribuidas a más de $500 \mathrm{~km}$ de los Alpes, al norte de la línea Ginebra-Le Havre (Petrequin et al. 2002; 2011b; Petrequin y Jeunesse, 1995), son anchas de sección delgada y color verde claro. Sin embargo, algunos autores (D'Amico et al. 1995; 2003; D’Amico, 2005; D'Amico y Starnini 2006), interpretan que su distribución sigue una curva de decrecimiento monótono en función a la distancia a la fuente o modelo down-the-line de Renfrew (1977).
El color y el tipo (Durringto/Puymirol) del hacha de la Colección Tubino parecen ubicarla cronológicamente a mediados/fines del V milenio a.n.e., en un segundo momento de dispersión de las hachas alpinas hacia Europa Occidental, coincidiendo con un cambio en el criterio de selección de materias primas (se prima el uso de jadeíta frente al dominio de las eclogitas y anfibolitas desde fines del VI milenio a.n.e.). Las jadeítas dominan el registro arqueológico (95\% del registro) al oeste de los Alpes hasta el golfo de Morbihan. Este dominio se extiende hacia Gran Bretaña, Irlanda y Alemania hacia fines del V milenio a.n.e. (Petrequin et al. 1997; 2002; 2011a; 2011b).

Desde finales del VI milenio a.n.e. hasta comienzos del III milenio a.n.e. los Alpes proporcionaron la materia prima de la mayoría de las hachas pulimentadas realizadas en jadeíta y eclogita del norte de Europa (Petrequin et al. 2008; 2011b). Estas hachas circularon a grandes distancias a lo largo de Europa Occidental, y en particular entre los Alpes, el Atlántico y el Mar del Norte, viajando desde los Alpes a Francia, Alemania, Luxemburgo, Bélgica, Holanda, Gran Bretaña (incluida Escocia), Irlanda y ocasionalmente hacia Escandinavia, República Checa, Eslovaquia, Austria, Croacia, el sur de Italia (Sicilia), España, Dinamarca y Bulgaria para cronologías que van desde el Neolítico a la Edad del Cobre, llegando a recorrer hasta $1700 \mathrm{~km}$ desde la fuente de aprovisionamiento (Venturino Gambari 1996; D'Amico 2005; D'Amico et al. 2003; Petrequin et al. 2005; 2008; 2011a; Tsonev 2008; Risch 2011; Leighton y Dixon 1992).

Petrequin et al. (2011b, 2002, 2005, 2008, 2006) proponen que las hachas alpinas comienzan a circular en dirección oeste a finales del VI milenio a.n.e., como herramientas puramente funcionales realizadas principalmente en eclogitas y anfibolitas, estando tan solo pulidas en el filo, limitando el uso de jadeítas a la producción de pequeñas hachas/cinceles y anillos. Durante la primera mitad del $\mathrm{V}$ milenio a.n.e. siguió predominando el uso de eclogitas, onfacitas y anfibolitas al mismo tiempo que surgían las grandes azuelas del tipo Bégude en el arco del piedemonte alpino en Italia y el piedemonte francés. A mediados del V milenio a.n.e. se dio un cambio en el criterio de selección de materias primas. Las jadeítas dominan el registro arqueológico (95\% del registro) al oeste de los Alpes hasta el golfo de Morbihan. Sin embargo, el norte de Europa y especialmente las llanuras del valle del Po parecen haber quedado fuera de este circuito de intercambio de grandes hachas de jadeíta, que se extendió hacia Gran Bretaña, Irlanda y Alemania hacia fines del $\mathrm{V}$ milenio a.n.e. En su viaje hacia la Bretaña las hachas 
habrían sufrido un proceso de selección -las largas y finas del tipo Durrington y Puymirol- que a una distancia de $500 \mathrm{~km}$ de la fuente habrían podido ser repulidas para ajustarse al tipo Altenstadt-Greenlaw. Cuando las hachas llegaron al golfo de Morbihan, a $1000 \mathrm{~km}$ de distancia de su origen, un buen número de ellas se repulirían una segunda vez para obtener hachas del tipo Tumiac y Carnac. Esto supone que algunas hachas habrían dejado de cumplir su principal cometido como herramientas para cumplir una función ritual, como objetos sagrados, que se depositan en tumbas de individuos excepcionales, como en la región de Carnac. Es más, ciertos tipos de hachas repulidas en el golfo de Morbihan, el tipo Carnac, se reintroducen dentro de los circuitos de circulación de hachas viajando hacia el noroeste de la península ibérica, Alemania, los Alpes, Italia y Croacia, llegando a recorrer hasta $2500 \mathrm{~km}$ (Petrequin et al. 2011b).

En la península ibérica el registro de hachas alpinas documentado hasta la fecha es extremadamente bajo. Tan solo se ha publicado un fragmento con filo de un hacha de jadeíta en Can Gafa (río Tenes, Barcelona) y dos hachas de jadeíta encontradas como parte del ajuar del sepulcro de fosa de la Bòbila d'en Joca (Ripollet, Barcelona), tan sólo $12 \mathrm{~km}$ al sur de Can Gafa (Risch y Martínez Fernández 2008: 51; Gibaja Bao 2005).

El inventario publicado por Pétrequin et al. (2012: Anexo 1, 720-721) incluye 4 hachas identificadas como jade en la costa mediterránea de la península ibérica: 2 en la Bòbila d'En Joca (Ripollet, Barcelona), 1 en la Bòbila Padró (Ripollet, Barcelona) y 1 en Bòbila Madural (Sant Quirce del Vallés, Barcelona). Por su parte, Fábregas et al. (2012: 1111 en adelante) refieren la existencia en la península ibérica de 9 hachas realizadas en Jade, las 4 de la costa mediterránea, además de 1 en Ourense y 4 más en la Meseta Norte, de las cuales la mayoría carecen de contexto arqueológico (procedentes de colecciones de museos o hallazgos aislados) y no han sido caracterizadas por métodos analíticos."

Desde un punto de vista cronológico, estos hallazgos parecen corresponder a contextos funerarios datados entre fines del V e inicios del IV milenio a.n.e. (Gibaja Bao 2004; 2005; Gibaja Bao et al. 2010). Desde el punto de vista geográfico, estas piezas se relacionarían con las hachas alpinas que circularon por el arco mediterráneo a fines del V milenio a.n.e. (Petrequin et al. 2011b, 2012 b) y procederían indudablemente de los Alpes tal y como confirma su caracterización como jadeíta (Risch 2011). Por tanto, la pieza de la Colección Tubino pasa a ser la cuarta entrada del exiguo catálogo de hachas de jade-jadeíta publicadas en la península ibérica, al tiempo que se convierte, con mucha diferencia, en la más meridional de ellas -además de una de la más alejada de las fuentes alpinas a escala europea $(c 1900 \mathrm{~km})$.

La presencia de esta pieza en el extremo meridional ibérico debe ser vista en el marco de la existencia de circuitos supra-regionales de intercambios de materias primas desde al menos el V-IV milenios a.n.e. En el Noreste ibérico, de donde proceden las tres piezas mencionadas, estos circuitos incluyen el intercambio de variscita de Can Tintorer (Gavá, Barcelona) hacia la Bretaña francesa (Querré et al. 2007), obsidianas sardas (Bosch et al. 2009, Terradas et al. 2014), sal de Cardona (Weller y Figuls 2007), jadeíta alpina (Risch 2011; Risch y Martínez Fernández 2008), fibrolita (Petrequin et al. 2011b), etc. Revisiones recientes han puesto de manifiesto la gran cantidad y diversidad de rocas raras y materias primas exóticas halladas en contextos funerarios del IV-II milenios a.n.e. en el sur de España, y que incluye cristal de roca, ámbar, variscita, marfil entre otros (Costa Caramé et al. 2011; Murillo-Barroso y Martinón-Torres, 2012; Schuhmacher et al., 2009, Banerjee et al., 2011). Los datos disponibles para el establecimiento de estos intercambios son de muy escasa calidad, dado que el número de dataciones radiocarbónicas obtenidas de los contextos en los que se han producido estos hallazgos es muy bajo. No obstante, incluso a pesar de la limitación que supone que se desconozca el contexto de la pieza en cuestión, los datos aportados en este estudio sobre el hacha de jade de la Colección Tubino contribuyen considerablemente a situar el contexto en el que esta línea de investigación debe enmarcarse, ampliando el rango y la escala geográfica y cronológica de dichas interacciones.

\section{Agradecimientos}

Apoyos recibidos para la realización del estudio: financiación MINECO HAR2013-34620.

\section{BIBLIOGRAFÍA}

Banerjee, A.; Dindorf, W.; Mikdad, A.; Reischmann, T. y Schuhmacher, T. X. (2011): "Die Elfenbeinfunde aus Kehf-el-Baroud (Ziaïda, Ben Slimane, Marokko) und die Frage des Nordafrikanischen Elefanten”. Madrider Mitteilungen 52: 113-138.

Belén Deamos, M. (2002): "Francisco María Tubino y la arqueología prehistórica en España”, en M. Belén 
Deamos y J. Beltrán Fortes (eds.), Arqueología Fin de Siglo. La Arqueología Española de la Segunda Mitad del Siglo XIX (I Reunión Andaluza de Historiografía Arqueológica). Spal Monografías III: 4360. Sevilla, Universidad de Sevilla.

Bosch, J.; Gibaja Bao, J. F. y Gratuze, B. (2009): “Estudi d'una peça neolítica d'obsidiana procedent de la mina 83 de Gavà: tipologia, funcionalitat i petrologia". Rubricatum 4. Intervencions Arqueologiques a les Mines de Gavà (Sector Serra de Les Ferreres). Anys 1998-2009: 133-137. Gavá, Museo de Gavá.

Chiari, G.; Compagnoni, R. y Giustetto, R. (1996a): "Use of non destructive X-Ray Diffraction Analyses for the study of green stone artifacts". TRACCE [Online] 5. http://www.rupestre.net/tracce/?p=1076.

Chiari, G.; Compagnoni, R.; Giustetto, R. y Ricq-De Bouard, M. (1996b): Metodi Archeometrici pero lo Studio dei Manufatti in Oietra Levigata. Le Vie Della Pietra Verde. Omega. Torino.

Compagnoni, R.; Rolfo, R.; Manavella, F. y Salusso, F. (2007): "Jadeitite in the Monviso meta-ophiolite, Piemonte Zone, Italian western Alps". Periodico di Mineralogia 76: 79-89.

Cooney, G. (2002): "So many shades of rock: colour symbolism and irish stones axeheads", en A. Jones y G. Macgregor (eds.), Colouring the Past. The Significance of Colour in Archaeological Research: 93-198. Oxford, Berg.

Costa Caramé, M. E.; García Sanjuán, L.; Murillo-Barroso, M.; Parrilla Giráldez, R. y Wheatley, D. W. (2011): "Artefactos elaborados en rocas raras en los contextos funerarios del IV-II milenios ANE cal ANE en el Sur de España: una revisión", en L. García Sanjuán, L.; C. Scarre y D. W. Wheatley (eds.), Exploring Time and Matter in Prehistoric Monuments: Absolute Chronology and Rare Rocks in European Megaliths. Proceedings of the 2nd EMSG Meeting (Seville, November 2008). Menga: Journal of Andalusian Prehistory Monographs 01: 253-293. Sevilla, Junta de Andalucía.

D'Amico, C. (2005): "Neolithic 'greenstone' axe blades from northwestern Italy across Europe: a first petrographic comparison". Archaeometry 47: 235-252.

D’Amico, C.; Campana, R.; Felice, G. y Ghedini, M. (1995): "Eclogites and jades as prehistoric implements in Europe. A case of petrology applied to cultural heritage". European Journal of Minera$\log$ 7: 29-42.

D’Amico, C. y Starnini, E. (2006): “Prehistoric polished stone artifacts in Italy: a petrographic and archaeological assessment", en M. Maggetti y B. Messiga (eds.), Geomaterials in Cultural Heritage: 257-272. London, Geological Society.

D’Amico, C.; Starnini, E.; Gasparotto, G. y Ghedini, M. (2003): "Eclogites, jades and other HP-metaophiolites employed for prehistoric polished stone implements in Italy and Europe". Periodico di Mineralogia 73: 17-42.

Damour, A. (1865): Sur la Composition des Haches en Pierre Trouvées dans les Monuments Celtiques et Chez les Tribus Sauvages. Tiré à part des comptesrendus de l'académie des Sciences, T. LXI, séances du 21 au 28 août 1865 .

Damour, A. (1881): "Nouvelles analyses sur la jadeite et sur quelques roches sodifieres". Bulletin de la Société Française de Minéralogie 4: 157-164.

Deer, W. A.; Howie, R. A. y Zussman, J. (1992): An Introduction to the Rock Forming Minerals. Harlow, Pearson.

Edwards, H. G. M. y Chalmers, J. M. (2005): Raman Spectroscopy in Archaeology and Art History. Lugar de edición, Springer-Verlag.

Errera, M. (2004): "Decouverte du premier gisement de jade-jadeite dans les Alpes (2004) Implications concernant plusieurs lames de hache néolithiques trouvées en Belgique et dans les régions limitrophes". Notae Prehistoricae 24: 191-202.

Errera, M.; Petrequin, P. y Petrequin, A. M. (2004): “De l'image a l'objet: determination de la provenance d'artefacts archaeologiques par spectroradiometrie en reflectance diffuse". Revue Française de Photogramétrie et de Télédétection 193: 27-38.

Fábregas Valcarcel, R.; de Lombeira Hermida, A. y Rodríguez Rellán, C. (2012): "Spain and Portugal: long chisels and perforated axes. Their context and distribution", en P. Petrequin; S. Cassen, M. Errera; L. Klassen, A. Sheridan y A.M. Petrequin (dirs.), JADE. Grandes haches alpines du Néolithique européen. $V^{E}$ et $I V^{E}$ millénaires av. J.-C.: 1111-1135. Charenton-lePont, Presses Universitaires de Franche-Comté.

Forde, D. C. (1930): “On the use of greenstones (jadeite, callais, etc.) in the megalithic culture of Brittany". The Journal of the Royal Anthropological 5. Institute of Great Britain and Ireland 60: 211-234.

García Sanjuán, L.; Vargas Jiménez, J. M.; Hurtado Pérez, V.; Ruiz Moreno, T. y Cruz-Auñón Briones, R. (eds.) (2012): El Asentamiento Prehistórico de Valencina de la Concepción (Sevilla): Investigación y Tutela en el 150 Aniversario del Descubrimiento de La Pastora. Sevilla, Universidad de Sevilla. 
Gendron, F.; Smith, D. C. y Gendron-Badou, A. C. (2002): "Discovery of jadeite-jade in Guatemala confirmed by non-destructive raman microscopy". Journal of Archaeological Science 29: 837-851. doi:10.1006/jasc.2001.0723

Gibaja Bao, J. F. (2004): "Prácticas funerarias durante el Neolítico en Cataluña". Mainake 26: 9-27.

Gibaja Bao, J. F. (2005): "Los enterramientos neolíticos del Noreste de la Península Ibérica”. Anthropologica et Prcehistorica 116: 165-178.

Gibaja Bao, J. F.; Majó, T.; Chambon, P.; Ruíz, J. y Subirà, M. E. (2010): "Prácticas funerarias durante el Neolítico. Los enterramientos infantiles en el noreste de la Península Ibérica". Complutum 21: $47-$ 68. http://revistas.ucm.es/index.php/CMPL/article/ viewFile/CMPL1010220047A/28830

Giustetto, R.; Chiari, G. y Compagnoni, R. (2008): “An easy non-invasive X-ray diffraction method to determine the composition of Na-pyroxenes from high-density 'greenstone' implements". Acta Crystallographica Section A 64: 161-168.

Hardin, C. L. y Maffoi, L. (1997): “Introduction", en C. L. Hardin y L. Maffoi (eds.), Colour Categories in Thought and Language: 1-18. Cambridge, Cambridge University Press.

Harrison, R. y Orozco Köhler, T. (2001): “Beyond characterisation. Polished stone exchange in the western Mediterranean 5500-2000 BC". Oxford Journal of Archaeology 20: 107-127.

Hatipoğlu, M.; Başevirgen, Y. y Chamberlain, S. C. (2012): "Gem-quality Turkish purple jade: geological and mineralogical characteristics". Journal of African Earth Sciences 63: 48-61. doi:10.1016/j.jafrearsci.2011.11.004

Huang, E. (1999): "Raman spectroscopic study of 15 gem minerals". Journal of the Geological Society of China 42: 301-318.

Jones, A. y Macgregor, G. (2002): "Introduction: Wonderful things: colour studies in archaeology from Munsell to materiality", en A. Jones y G. Macgregor (eds.), Colouring the Past. The Significance of Colour in Archaeological Research: 1-22. Oxford, Berg.

Leighton, R. y Dixon, J. E. (1992): "Jade and greenstone in the prehistory of Sicily and southern Italy". Oxford Journal of Archaeology 11: 179-200.

Lillios, K. T. (1997): "Amphibolite tools of the Portuguese Copper Age (3000-2000 B.C.): A geoarchaeological approach to prehistoric economics and symbolism". Geoarchaeology: An International Journal 12: 137-163.
Morten, L.; Bargossi, G. M.; Martínez, J. M.; Puga, E. y De Federico, A. D. (1987): "Metagabbro and associated eclogites in the Lubrin area, Nevado-Filabride Complex, Spain." Journal of Metamorphic Geology 5: 155-174.

Murillo-Barroso, M. y Martinón-Torres, M. (2012): "Amber sources and trade in the prehistory of the Iberian peninsula". European Journal of Archaeology 15 (2): 187-216. DOI 10.1179/1461957112Y. 0000000009

Petrequin, P.; Cassen, S.; Croutsch, C. y Weller, O. (1997): "Haches alpines et baches carnaceennes dans l'Europe du Ve millenaire". Notae Praehistoricae: $135-150$.

Petrequin, P.; Cassen, S.; Gauthier, E.; Klassen, L.; Paillier, Y. y Sheridan, A. (2012): “Typologie, chronologie et répartition des grandes haches alpines en Europe occidentale", en P. Petrequin; S. Cassen, M. Errera; L. Klassen, A. Sheridan y A.M. Petrequin (dirs.), JADE. Grandes haches alpines du Néolithique européen. $V^{E}$ et IV millénaires av. J.-C.: 574-727. Charenton-lePont, Presses Universitaires de Franche-Comté.

Petrequin, P.; Cassen, S. y Errera, M. (2002): "La valorisation sociale des longues haches dans l'Europe Néolithique", en J. Guilaine (ed.), Matériaux, Productions, Circulations du Neolithique à l'Age du Bronze: 67-97. París, Errance.

Petrequin, P.; Errera, M.; Cassen, S.; Billand, G.; Colas, C.; Marechal, D. y Prodeo, F. (2005): "Des Alpes italiennes à l'Atlantique au Ve millénaire. Les quatre grandes haches polies de Vendeuil et Maizy (Aisne), Brenouille (Oise)". Revue Archéologique de Picardie 22: 75-104.

Petrequin, P.; Errera, M.; Cassen, S.; Gauthier, E.; Hovorka, D.; Klassen, L. y Sheridan, J. A. (2011a): "From Mont Viso to Slovakia: the two axeheads of Alpine jade from Golianovo". Acta Archaeologica Academiae Scientiarum Hungaricae 62: 243-268.

Petrequin, P., Errera, M., Martin, A., Fábregas Valcarce, R., y Vaquer, J. (2012b): “Les Haches En Jades Alpins Pendant Les Ve et IVe Millénaires. L'exemple de l'Espagne et Du Portugal Dans Une Perspective Européenne." Rubricatum: Revista Del Museu de Gavà 5: 213-22.

Petrequin, P.; Errera, M.; Petrequin, A. M. y Allard, P. (2006): "The Neolithic quarries of Mont Viso, Piedmont, Italy: initial radiocarbon dates". European Journal of Archaeology 9: 7-30. doi: $10.1177 / 1461957107077703$.

Petrequin, P. y Jeunesse, C. (1995): La Hache de Pierre. Carrieères Vosgiennes et Échanges de Lames 
Polies Pendant le Néolithique (5400-2100 av. J.C.). Paris, Errance.

Petrequin, P.; Sheridan, A.; Cassen, S.; Errera, M.; Gauthier, E.; Klassen, L.; Le Maux, N. y Pailler, Y. (2008): "Neolithic Alpine axeheads, from the Continent to Great Britain, the Isle of Man and Ireland", en H. Fokkens; B. J. Coles; A. L. Van Gijn; J. P. Kleijne; H.H. Ponjee y C. G. Slappende (eds.), Between Foraging and Farming: An Extended Broad Spectrum of Papers Presented to Leendert Louwe Kooijmans. Analecta Praehistorica Leidensia 40: 247-260. Leiden, Leiden University.

Petrequin, P.; Sheridan, A.; Cassen, S.; Errera, M.; Gauthier, E.; Klassen, L.; Le Maux, N.; Petrequin, A. M. y Rossy, M. (2011b): "Eclogite or jadeite: The two colours involved in the transfer of alpine axeheads in western Europe", en V. Davis y M. R. Edmonds (eds.), Stone Axe Studies III. Spain: 5582. Oxford, Oxbow Books.

Pin, C. y Vielzeuf, D. (1988): "Les granulites de hautepression d'Europe moyenne temoins d'une subduction eo-hercynienne. Implications sur l'origine des groupes leptyno-amphiboliques". Bulletin de la Société Géologique de France 8: 13-20.

Querré, G., Herbault, F. y Calligaro, T. (2007): “Long distance transport of Neolithic variscite ornaments along the European Atlantic are demonstrated by PIXE analysis". Proceedings of the XI International Conference on PIXE and its Analytical Applications Puebla, Mexico, May 25-29, 2007: 381-384. México DF, UNAM.

Renfrew, C. (1977): “Alternative models for exchange and spatial distribution", en T. K. Earle y K. L. Erickson (eds.), Exchange Systems in Prehistory: 71-90. New York, Academic Press.

Ricq-De-Bouard, M. (1993): Trade in Neolithic Jadeite Axes from the Alps: New Data. Trade and Exchange in Prehistoric Europe. Exeter, Oxbow Books.

Ricq-De-Bouard, M.; Compagnoni, R.; Desmons, J. y Fedele, F. (1990): "Les roches alpines dans l'outillage poli neolithique de la France mediterranenne". Gallia Préhistoire 32: 125-149.

Ricq-De-Bouard, M. y Fedele, F. G. (1993): "Neolithic rock resources across the western alps: circulation data and models". Geoarchaeology 8: 1-22.

Risch, R. (2011): "Social and economic organisation of stone axe production and distribution in the western Mediterranean", en V. Davis y M. R. Edmonds (eds.), Stone Axe Studies III. Spain: 100-118. Oxford, Oxbow Books.
Risch, R. y Martínez Fernández, F. (2008): “Dimensiones naturales y sociales de la producción de hachas de piedra en el noreste de la Península Ibérica". Trabajos de Prehistoria 65 (1): 47-71. http://tp.revistas. csic.es/index.php/tp/article/viewFile/135/136

Ruiz Moreno, T. (2012): "La Pastora (Valencina de la Concepción, Sevilla): una historia desconocida", en L. García Sanjuán; J. M. Vargas Jiménez; V. Hurtado Pérez; T. Ruiz Moreno y R. Cruz-Auñón Briones (eds.), El Asentamiento Prehistórico de Valencina de la Concepción (Sevilla): Investigación y Tutela en el 150 Aniversario del Descubrimiento de La Pastora: 201-218. Sevilla, Universidad de Sevilla.

Schuhmacher, T. X.; Cardoso, J. L. y Banerjee, A. (2009): "Sourcing African ivory in Chalcolithic Portugal". Antiquity 83: 983-997.

Smith, D. C. (2005a): "Case Study: Mesoamerican Jade", en H. G. M. Edwards y J. M. Chalmers (eds.), Raman Spectroscopy in Archaeology and Art History: 412426. Cambridge, Royal Society of Chemistry.

Smith, D. C. (2005b): "Overview: jewellery and precious stones", en H. G. M. Edwards y J. M. Chalmers (eds.), Raman Spectroscopy in Archaeology and Art History: 335-378. Cambridge, Royal Society of Chemistry.

Smith, D. C. y Gendron, F. (1997): “Archaeometric application of the Raman microprobe to the nondestructive identification of two pre-Columbian ceremonial polished 'greenstone' axe-heads from Mesoamerica". Journal of Raman Spectroscopy 28: 731-738.

Smith, G. D. y Clark, R. J. H. (2004): "Raman microscopy in archaeological science". Journal of Archaeological Science 31: 1137-1160. doi:10.1016/j. jas.2004.02.008

Terradas, X.; Gratuze, B.; Bosch, J.; Enrich, R.; Esteve, X.; Oms, F.X. y Ribé, G. (2014): "Neolithic Diffusion of Obsidian in the Western Mediterranean: New Data from Iberia." Journal of Archaeological Science 41: 69-78. doi:10.1016/j.jas.2013.07.023

Thirault, E. (2005): "The politics of supply: the Neolithic axe industry in Alpine Europe". Antiquity 79: 34-50.

Tsonev, T. (2008): "The social dimension of distance in prehistory: a jadeite axe case from Bulgaria", en R. I. Kostov; B. Gaydarska y M. Gurova (eds.), Geoarchaeology and Archaeomineralogy. Proceedings of the International Conference (29-30 October, 2008, Sofia): 60-62. Sofia, St. Ivan Rilski Publishing House.

Tubino y Oliva, F. M. (1868a): "Monumento Prehistórico de Castilleja de Guzmán". Gaceta de Madrid 23 de Marzo 1868: 49-59. 
Tubino y Oliva, F. M. (1868b): “Monumento Prehistórico de Castilleja de Guzmán”. Estudios Prehistóricos: 49-58. Madrid, Oficinas de la Revista de Bellas Artes.

Tubino y Oliva, F. M. (1876): "Los monumentos megalíticos de Andalucía, Extremadura y Portugal y los aborígenes ibéricos". Museo Español de Antigüedades 7: 303-364.

Tuncer Arslanlar, Y.; Garcia-Guinea, J.; Kibar, R.; Çetin, A.; Ayvacikli, M. y Can, N. (2011): "Luminescence behavior and Raman characterization of jade from Turkey". Applied Radiation and Isotopes 69: 1299-1306. doi:10.1016/j.apradiso.2011.05.011
Vargas Jiménez, J. M.; Meyer, C. y Ortega Gordillo, M. (2012): "El tholos de La Pastora y su entorno: el sector oriental del yacimiento de Valencina de la Concepción (Sevilla) a través de la geofísica". Menga. Revista de Prehistoria de Andalucía 3: 121-140.

Venturino GambarI, M. (1996): Le Vie della Pietra Verde. La Industria Litica Levigata nella Preistoria dell'Italia Settentrionale. Torino, Omega.

Weller, O. y Figuls, A. (2007): "L'intercanvi com a dinamitzador econòmic del Neolític mitjà a Catalunya i la primera explotació minera de sal gemma d'Europa: la vall Salina de Cardona (Barcelona)". Cota Zero 22: 101-111. 